"Adding value to satisficing decisions using TOPSIS in service provider selection problems"

\section{AUTHORS}

ARTICLE INFO

DOI

RELEASED ON

JOURNAL

FOUNDER
Solly Matshonisa Seeletse

Solly Matshonisa Seeletse (2016). Adding value to satisficing decisions using TOPSIS in service provider selection problems. Environmental Economics, 7(1), 34-39. doi: 10.21511/ee.07(1).2016.04

http://dx.doi.org/10.21511/ee.07(1).2016.04

Thursday, 24 March 2016

"Environmental Economics"

LLC "Consulting Publishing Company "Business Perspectives"

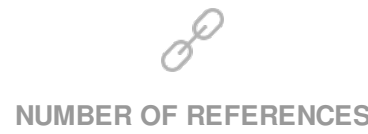

0

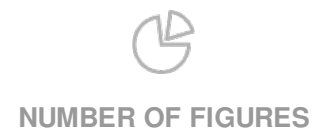

0

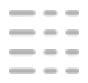

NUMBER OF TABLES

0

(C) The author(s) 2022. This publication is an open access article. 
Solly Matshonisa Seeletse (South Africa)

\title{
Adding value to satisficing decisions using TOPSIS in service provider selection problems
}

\begin{abstract}
Selection processes of credible candidates in competitions are often flawed. The flaws may be deliberate when there is corruption. In other cases the flaws occur because of the decision makers' inadequacies. Many competitors do their best in developing exceptional proposals, but unfairness of the decision makers undermines these efforts. Ideally, undeserving candidates should be disqualified, and deserving ones be allowed to contest. Systematic methods should be used in the proposal evaluation, and the process should be verifiable. This paper discusses scientific methods proposed for use to select a criterion-based worthy competitor in service provider selection problems. The method is a technique for order preference by similarity to ideal solution (TOPSIS). TOPSIS is a mathematically-derived statistical method useful to offset the biases in the selection process. Features that address both added value and reduced costs are incorporated in the TOPSIS selection process. A numerical example is included to demonstrate TOPSIS fortes.
\end{abstract}

Keywords: attributes, criteria, satisficing, service provider selection, scientific method. JEL Classification: C1, C4, C5, C6.

\section{Introduction}

Client companies who outsource some of their organizations' activities do not always select the best expert companies for the tasks they outsource. Officials involved in the selection of companies to which they wish to outsource might be favored with no merit reason (such as corruption), or due to lacking the appropriate knowledge to select a worthy company capable of delivering the best service. Even when the chosen company meets the minimum criteria for the tasks at hand, it is advisable to attempt to select a company that can also add value to the solution. Selecting the candidate who is not the best undermines the idea of outsourcing which is done to involve experts. It also undermines the proposal development for diligent preparation to do best in the tasks to be outsourced. TOPSIS (technique for order preference by similarity to ideal solution) is a scientific method that can assist to optimize the decision problems of selecting leading expert companies to which outsourcing can be directed. It can identify weak alternatives, thus ensuring that no mediocre service providers are selected. It also identifies leading candidates, thus ensuring that only top candidates are selected. It also identifies ideal performance, and the top performers. A warning by Triantaphyllou (2000) is that decision problems based on many variables and many conditions are easy for cheats to influence. However, according to Assari, Mahesh and Assari (2012), TOPSIS is a scientific method that can deal with any situation requiring problem solving even in multifaceted settings. Chen, Tzeng and Ding (2003) counsel that decision prob-

(c) Solly Matshonisa Seeletse, 2016.

Solly Matshonisa Seeletse, Professor of Operations Research, Department of Statistics and Operations Research, Sefako Makgatho Health Sciences University, South Africa. lems addressed using subjective methods criteria enable favoritism, and are often controversial. These methods lead to mediocre results, and to lack of development. Members involved in the same team to make a selection may end up giving varying details for the same procedures when subjectivity and bias dominate the procedure. Wang and Lee (2007) emphasize use of scientific methods that can reduce and sometimes eliminate human bias to defy subjectivity and favoritism.

This paper presents TOPSIS in the selection process to identify a competitor who satisfies the minimum criteria, who can also perform tasks better than any other company who also meets the minimum criteria. TOPSIS supports impartiality in selection problems, and provides a method to reduce bias. It reveals the theoretically best desirable company for competitor selection. It also identifies the worst possible companies not desirable in the selection, and assists in alienating the selection from such undesirable candidates. Then, in acceptance of lack of possibility to reach the ideal, TOPSIS selects the best compromize closest to the ideal. This is the case for ensuring that the minimum criteria are met, and the selection picks the leading candidate who can do the work better than any other competitor who also meets the minimum criteria. Deng, Yeh and Willis (2000) explain that TOPSIS ensure that the final candidate winning the selection meets all the minimum requirements (said to be satisficing) (i.e. has the uppermost tally for order of preference; bear best resemblance to the ideal features; and simultaneous contrasts the undesirable features them).

\section{TOPSIS}

The method illustrated in this paper is the "technique for order preference by similarity to ideal solution' (TOPSIS). TOPSIS is as a mathematical index value 
used to compare different options (Köksalan, Wallenius \& Zionts, 2011). It is obtained from metric measures such that the chosen solution is 'as close to the positive ideal solution (PIS) as possible and as far away from the negative ideal solution (NIS) as possible'. TOPSIS development is usually based on many attributes ( $m$, say), setting a stage of Multiattribute Decision Making (MADM). The different attributes may differ in importance. Weights are then used to indicate the differences in the value of importance for each attribute. This is discussed in section 2.

1.1. Matrix system for TOPSIS. Quantitative procedures involving many variables are easily presented using a matrix. Define the $n \times m$ matrix $X$ with columns signifying the $m$ criteria and the $n$ rows indicating the options in a competition. This matrix is the decision matrix (Deng et al., 2000) having the form:

$$
X=\left[x_{i j}\right], i=1,2, \ldots, n ; j=1,2, \ldots, m .
$$

Naturally, different attributes appear in different units. On the other hand, systematic methods are applicable only on units that are not comparable. It is then crucial to transform values into comparable units (Assari, Mahesh and Assari, 2012). The vector normalization of matrix elements into performance indices is defined as:

$$
p_{i j}=\frac{x_{i j}}{\sum_{s=1}^{n} \sum_{j=1}^{m} x_{s m}} \text {. }
$$

These performance indexes have two properties:

$$
\begin{aligned}
& \text { 1. } \quad 0 \leq p_{i j} \leq 1 \text {; and } \\
& \text { 2. } \quad \sum_{i=1}^{n} \sum_{j=1}^{m} p_{i j}=1 .
\end{aligned}
$$

From this, the performance index matrix $P$ is defined as:

$$
P=\left[p_{i j}\right], i=1,2, \ldots, n ; j=1,2, \ldots, m .
$$

1.2. Optimization. Suppose that the decision problem consists of a total of $n$ decision variables, $k$ constraints and $m$ attributes. The term "optimize" implies "minimize" when the cost attributes are involved, and implies "maximize" on benefit attributes (Rezaei, 2015). Thus, "optimum" on benefit attributes implies maximum, and on cost attributes it refers to minimization. Let $X$ denote a set of suitable constraining functions $g_{h}(x), h=1$, $2, \ldots, k ; f_{j}(x)$ the benefit objective for maximization, $j$ $\in J$; and $f_{i}(x)$ the cost objective for maximization, $i$ $\in I$. Ozelkan and Duckstein (2000) express MADM optimization problems using the method:

\section{Optimize}

$\left[f_{1}(x) \quad f_{2}(x) \cdot \ldots \cdot f_{m}(x)\right]$

such that

$x \in X=\left\{x: g_{h}(x)\{\leq,=, \geq\} 0 ; h=1,2, \ldots, k\right\}$.

1.3. TOPSIS reference points. The reference points as defined by Bragge, Korhonen, Wallenius and Wallenius (2010) from equation (4) for $j=1,2$, ... $m$ are as follows:

$f_{t}^{b}=\left\{\begin{array}{l}\max _{x \in X} f_{j}(x) \text { for all } j \in J \\ \min _{x \in X} f_{i}(x) \text { for all } i \in I\end{array}\right.$,

and

$f_{t}^{w}=\left\{\begin{array}{l}\min _{x \in X} f_{j}(x) \text { for all } j \in J \\ \max _{x \in X} f_{i}(x) \text { for all } i \in I\end{array}\right.$.

Let $f^{b}=\left(f_{1}^{b}, f_{2}^{b} \ldots f_{n}^{b}\right)$ be the solution to equation (5) consisting of individual best feasible solutions for all objectives, and $f^{w}=\left(f_{1}^{w}, f_{2}^{w} . . f_{n}^{w}\right)$ be the solution of equation (6) consisting of individual worst feasible solutions for all objectives. Assari, Mahesh and Assari (2012) consider $f^{b}$ as the PIS and $f^{v}$ as the NIS. Chen et al. (2003) indicate that these solutions are always out of the feasible regions of (4a) and (4b). Hence, this impediment is addressed by a compromise solution discussed later.

1.4. Philosophy for application of TOPSIS. TOPSIS is a data reduction descriptive measure. It reduces a multi-dimensional objective space to a twodimensional objective space where the two dimensions are the distance from PIS and the distance from NIS. It then eradicates the intrinsic differences in units between the original variables. According to Deng et al. (2000), there is a "conflict" between the distances from PIS and that from NIS. The conflict is due to the impracticality to concurrently obtain the shortest distance from PIS and the longest distance from NIS. Then, compromize criteria were introduced to replace the ideal ones. The compromize criteria are such that "the shortest distance from PIS" is replaced by "as close to PIS as possible"; and "the farthest distance from NIS" is replaced by "as far away from NIS as possible".

1.5. Value. Decisions are usually made on the argument that the selection made is satisficing, which means satisfying the minimum requirements. The question of 'added value' often implies optimally meeting all the requirements, with some additional 
work beyond the requested level, with waste reduced and at the lowest price to the client without disadvantages to the service provider's profits (Seeletse, 2015). This paper uses only the top few relevant criteria for service provider selection to disable waste interference. It also selects only the satisficing service provider, but focuses on closeness to fulfilment of maximum requirements since in practice the ideal case of maximum fulfilment of requirements is non-existent.

\section{Weights}

Attributes are selected because of their importance. However, in many instances, the levels of importance differ. Weights are able to describe the levels of relative importance of the different variables, or attributes. Larger weights signify higher importance. In this paper the weights $w_{j}$ for $j=1,2, \ldots, m$ designate the relative worth or importance of attributes. Many ways are used to derive weights. This paper uses an entropy measure to derive the weights.

2.1. Entropy. Entropy is a measure derived from the physical sciences using mathematical methods to measure the amount of disorder in a system (Deng et al., 2000). Thus, in statistical mechanics, entropy is used essentially to measure the number of ways in which a system is arranged. Jungermann (2006) explains the entropy as a measure of "disorder" implying that higher entropy indicates a higher disorder. Atkins and De Paula (2006) explain that entropy quantifies the information contained in a message in the sense of an expected value. Let "ln" denote the natural logarithm to the base e. Define $k=(\ln n)^{-1}$. Deng et al. (2000) define the entropy measure $e_{j}$ as:

$$
e_{j}=-k \sum_{i=1}^{n} p_{i j} \ln p_{i j}, \quad j=1,2, \ldots ., m .
$$

2.2. Degree of divergence. The degree of divergence $\left(d_{j}\right)$ represents the inherent contrast of the attribute $X_{j}$ (Deng et al., 2000). It is defined (and thus calculated) as:

$$
d_{j}=1-e_{j}, \quad j=1,2, \ldots, m .
$$

Performance ratings that diverge more for the attribute $X_{j}$ influence the $d_{j}$ to become larger, which then implies that the attribute $X_{j}$ is more important for the problem at hand (Baierlein, 2003). Logic of the definition of entropy means that $d_{j}$ is a measure of the amount of order of an attribute. Further rationality about $d_{j}$ is that an attribute is not important for a specific problem if in that problem all the options have equal perfor- mance ratings for that specific attribute. Thus, if all performance ratings for that attribute are equal, the attribute can be removed for the situation on which a decision is based as it transmits no useful information in rating.

2.3. Weights derivation. The weights derived from entropy (through $d_{j}$ ) for each attribute are given by Deng et al. (2000) as:

$$
w_{j}=\frac{d_{j}}{\sum_{k=1}^{n} d_{k}}, \quad j=1,2, \ldots, m .
$$

\section{Computing the TOPSIS index}

It was stated earlier that PIS $f^{b}$ and the NIS $f^{w}$ solutions always fall outside the feasible region of (4a) and (4b). Thus, TOPSIS is a compromise value obtained from some rational blend of NIS and PIS. The TOPSIS index is created from distance measures (Deng et al., 2000; Ozelkan \& Duckstein, 2000) as follows:

$$
\begin{array}{ll}
P_{i}=\left[w_{j}\left(f_{j}^{b}-x_{i j}\right)^{2}\right]^{\frac{1}{2}}, & i=1,2, \ldots, n . \\
N_{i}=\left[w_{j}\left(x_{i j}-f_{j}^{w}\right)^{2}\right]^{\frac{1}{2}}, & i=1,2, \ldots, n .
\end{array}
$$

The TOPSIS index is an overall performance measure calculated for each alternative $A_{i}, i=1,2$, $\ldots, n$ by:

$T_{i}=\frac{N_{i}}{P_{i}+N_{i}}, i=1,2, \ldots, n$.

Larger $T_{i}$ index values indicate higher performance. When ranking TOPSIS indices, values from largest to smallest indices are $T_{(1)}, T_{(2)}$, . ., $T_{(n)}$, and the corresponding alternatives then appear as $A_{(1)}, A_{(2)}, \ldots, A_{(n)}$.

\section{Properties of $e_{j}, d_{j}, w_{j}$ and $T_{j}$}

Important results of the entropy measure, degree of divergence and the weights used in deriving both these measures are as follows:

Result 1: $\quad 0 \leq e_{j} \leq 1, \quad j=1,2, \ldots, m$.

Result 2: $\quad 0 \leq d_{j} \leq 1, \quad j=1,2, \ldots, m$.

Result 3: $\quad 0 \leq w_{j} \leq 1, \quad j=1,2, \ldots, m$.

Result 4: $\quad 0 \leq T_{i} \leq 1, \quad i=1,2, \ldots, n$.

Proofs of these properties can be found in Seeletse (2014). 


\section{Numerical exercise}

5.1. Data matrix. The author of this paper was tasked to develop merit scores from the marks awarded on the proposals submitted by small entreprizes for a mini-project that was required in a government department in South Africa. A matrix of the marks was used to develop merit indices. It was supplied as:

\begin{tabular}{|c|c|c|c|c|}
\hline$A 1$ & {$[61$} & 62 & 61 & 74 \\
\hline 42 & 46 & 51 & 42 & 47 \\
\hline & 52 & 60 & 50 & 53 \\
\hline & 81 & 57 & 92 & 62 \\
\hline & 51 & 66 & 74 & 63 \\
\hline & 30 & 38 & 65 & 49 \\
\hline & 61 & 50 & 53 & 62 \\
\hline & 94 & 51 & 40 & 49 \\
\hline & 40 & 52 & 64 & 66 \\
\hline
\end{tabular}

This matrix denotes that nine alternative companies competed for the outsourcing job. They were judged on five benefit criteria $(\mathrm{C} 1, \mathrm{C} 2, \mathrm{C} 3, \mathrm{C} 4, \mathrm{C} 5)$ given on the five columns of the matrix. As a decision matrix it is presented as:

$$
X=\left[\begin{array}{lllll}
61 & 62 & 61 & 74 & 71 \\
46 & 51 & 42 & 47 & 44 \\
52 & 60 & 50 & 53 & 50 \\
81 & 57 & 92 & 62 & 71 \\
51 & 66 & 74 & 63 & 64 \\
30 & 38 & 65 & 49 & 30 \\
61 & 50 & 53 & 62 & 64 \\
94 & 51 & 40 & 49 & 53 \\
40 & 52 & 64 & 66 & 62
\end{array}\right]
$$

The criteria are all benefit criteria, and are few. Face value observation shows companies A1, A4 and A5 to be outstanding "high performers" while A2 and A6 are "low performers".

5.2. Performance matrix. Using equation (2), the performance matrix is:

$P=\left[\begin{array}{ccccc}0.0237 & 0.0240 & 0.0237 & 0.0287 & 0.0275 \\ 0.0178 & 0.0198 & 0.0163 & 0.0182 & 0.0171 \\ 0.0202 & 0.0233 & 0.0194 & 0.0206 & 0.0194 \\ 0.0314 & 0.0221 & 0.0357 & 0.0240 & 0.0275 \\ 0.0198 & 0.0256 & 0.0287 & 0.0244 & 0.0248 \\ 0.0116 & 0.0147 & 0.0252 & 0.0190 & 0.0116 \\ 0.0237 & 0.0194 & 0.0206 & 0.0240 & 0.028 \\ 0.0365 & 0.0198 & 0.0155 & 0.0190 & 0.0206 \\ 0.0155 & 0.0202 & 0.0248 & 0.0256 & 0.0240\end{array}\right]$

5.3. Weights. $n=9, k=(\ln n)^{-1}=0.4551$,

$e_{j}=-k \sum_{i=1}^{n} p_{i j} \ln p_{i j}, j=1,2, \ldots, 5$.

\begin{tabular}{|l|l|l|l|l|}
\hline 0.3419 & 0.3312 & 0.3559 & 0.3501 & 0.3407 \\
\hline
\end{tabular}

$d_{j}=1-e_{j}, \quad j=1,2, \ldots, 5$.

\begin{tabular}{|l|l|l|l|l|}
\hline 0.6581 & 0.6688 & 0.6441 & 0.6499 & 0.6593 \\
\hline
\end{tabular}

$w_{j}=\frac{d_{j}}{\sum_{k=1}^{n} d_{k}}, j=1,2, \ldots, 5$.

\begin{tabular}{|l|l|l|l|l|}
\hline 0.2006 & 0.2039 & 0.1964 & 0.1981 & 0.2010 \\
\hline
\end{tabular}

PIS: $\quad f^{p}=\left(\begin{array}{lllll}94 & 66 & 92 & 74 & 71\end{array}\right)$

NIS: $f^{n}=\left(\begin{array}{lllll}30 & 38 & 40 & 47 & 30\end{array}\right)$

$P_{i}=\left[w_{j}\left(f_{j}^{b}-x_{i j}\right)^{2}\right]^{\frac{1}{2}}, i=1,2, \ldots, 9$

\begin{tabular}{|l|l|l|l|l|l|l|l|l|}
\hline 28.79 & 48.17 & 41.23 & 24.92 & 31.33 & 53.36 & 36.47 & 41.22 & 38.50 \\
\hline
\end{tabular}

According to order of highest desirability as identified by smallest distances, they are $P_{4}, P_{1}, P_{5}, P_{7}$, $P_{9}, P_{8}, P_{3}, P_{2}, P_{6}$, and these identify the corresponding desirable options.

$$
\begin{aligned}
& N_{i}=\left[w_{j}\left(x_{i j}-f_{j}^{w}\right)^{2}\right]^{\frac{1}{2}}, \quad i=1,2, \ldots, 9 \\
& \begin{array}{|l|l|l|l|l|l|l|l|l|}
\hline 36.28 & 16.31 & 23.33 & 44.52 & 34.46 & 18.19 & 28.57 & 33.30 & 28.54 \\
\hline
\end{array}
\end{aligned}
$$

According to order of highest undesirability using smallest distances, they are $N_{2}, N_{6}, N_{3}, N_{9}, N_{7}$, $N_{8}, N_{5}, N_{1}, N_{4}$, and these identify the corresponding undesirable options.

$T_{i}=\frac{N_{i}}{P_{i}+N_{i}}, i=1,2, \ldots, 9$

\begin{tabular}{|l|l|l|l|l|l|l|l|l|}
\hline 0.558 & 0.253 & 0.361 & 0.641 & 0.524 & 0.254 & 0.439 & 0.447 & 0.426 \\
\hline
\end{tabular}

Ordering the scores from largest to smallest:

$T_{4}, T_{1}, T_{5}, T_{8}, T_{7}, T_{9}, T_{3}, T_{6}, T_{2}$.

5.4. Summary of results. Order according to high desirability: $P_{4}, P_{1}, P_{5}, P_{7}, P_{9}, P_{8}, P_{3}, P_{2}, P_{6}$ (PIS).

Order according to most undesirability: $N_{2}, N_{6}$, $N_{3}, N_{9}, N_{7}, N_{8}, N_{5}, N_{1}, N_{4}$ (NIS).

Order according to closest to desired ideal: $T_{4}, T_{1}$, $T_{5}, T_{8}, T_{7}, T_{9}, T_{3}, T_{6}, T_{2}$ (TOPSIS).

NIS identified the most undesirable solutions by picking the most undesired results while PIS identi- 
fied the most desirable ones. Regularity is demonstrated where the worst feasible result is the least ranked according to the PIS. Also, TOPSIS relegates the solutions identified as NIS, and then also identified the desirable solutions that were also picked by PIS. Option A2, A6 and A3 are undesirable according to all the methods. Also, from all the three methods, A4 appears as the best, followed by A1 and A5.

\section{Discussion}

TOPSIS compares the candidates in the competition. Use of the numerical exercise in this paper illustrates TOPSIS strengths. It showed that even though TOPSIS is required basically for complex approaches to waste reduction and value adding, its fundamental philosophy is to perform in the way the human mind logic appeals in reducing waste and increasing value. It identified weak competitors that were not satisficing. The weakest losers in the competition were ranked according to relative weakness using NIS. The identification was consistent with the use of NIS. Similarly, strong candidates were identified and ranked with ease.

The desirable competitors selected using PIS were ranked by merit. Then TOPSIS also selected and ranked the leading ones. Thus, satisficing candidates were identified and those not meeting the minimum requirements were disqualified. This assisted in reducing waste. The remaining candidates qualified to be the preferred service providers. However, once NIS has assisted in identifying candidates for deletion, PIS identifies satisficing ones. The complementary part of NIS and PIS is that while NIS ranks candidates of weakest, PIS ranks the toughest ones. Coincidence is that the relegated ones in NIS are the weakest in PIS. This indicates reliability of selection in identification of meritorious candidates by TOPSIS and its subsidiary methods. In the end the con- ciliation solution provided by TOPSIS supports the elimination of candidates by NIS that were shown to be the weakest and also approves the PIS identified candidates. TOPSIS finalizes the process by selecting the best among the leading competitors.

\section{Recommendation}

This study discourages subjective methods in applications where meritorious decisions are required. Subjective methods undermine positive development as they easily enable mediocrity to contaminate good work. Hence, the study recommends that unfair approaches should not be considered as rational decision making.

Furthermore, the study recommends use of scientific methods in all selection where merit is a necessary criterion. A method such as NIS should be used to ensure that weak candidates are disqualified. Also, PIS or equivalent method should always be available to corroborate selection of meritorious candidates.

\section{Conclusion}

TOPSIS is ideal for complex projects where many criteria are involved in the selection and many competitors are compared. TOPSIS approach contains NIS and PIS to assist in removing waste and adding value. The final TOPSIS step is a compromise that confirms and supports NIS and PIS with waste removal and adding value, and selecting the final solution that is closest to the ideal one.

\section{Acknowledgements}

The department which granted permission to use the data for this research strictly gave permission provided that neither its name nor identities of parties were made. However, it is fully acknowledged. Also, the Department of Statistics and Operations Research of Sefako Makgatho Health Sciences University is acknowledged in supporting this paper.

\section{References}

1. Assari, A., Mahesh, T. \& Assari, E. (2012). Role of public participation in sustainability of historical city: Usage of TOPSIS method, Indian Journal of Science and Technology, 5(3), pp. 2289-2294.

2. Atkins, P. \& De Paula, J. (2006). Physical chemistry, $8^{\text {th }}$ edition. Oxford University Press.

3. Baierlein, R. (2003). Thermal physics. London: Cambridge University Press.

4. Bragge, J., Korhonen, P., Wallenius, H. \& Wallenius, J. (2010). Bibliometric analysis of multiple criteria decision making/multiattribute utility theory. IXX International MCDM Conference Proceedings, (Eds.) M. Ehrgott, B. Naujoks, T. Stewart, and J. Wallenius, Springer, 634, pp. 259-268.

5. Chen, M.F., Tzeng, G.H. \& Ding, C.G. (2003). Fuzzy MCDM approach to select service provider, IEEE International Conference on Fuzzy Systems, pp. 572-577.

6. Deng, H., Yeh, C.H. \& Willis, R.J. (2000). Inter-company comparison using modified TOPSIS with objective weights, Computers and Operations Research, 27, pp. 963-973.

7. Jungermann, A.H. (2006). Entropy and the shelf model: A quantum physical approach to a physical property, Journal of Chemical Education, 83, pp. 1686-1694.

8. Köksalan, M., Wallenius, J. \& Zionts, S. (2011). Multiple criteria decision making: From early history to the $21^{\text {st }}$ century. Singapore: World Scientific. 
9. Ozelkan, E.C. \& Duckstein, L. (2000). Multi-objective fuzzy regression: a general framework, Computers and Operations Research, Special Issue on Artificial Intelligence and Decision Support with Multiple Criteria, 27 (7 \& 8) pp. 635-652.

10. Rezaei, J. (2015). Best-worst multi-criteria decision-making method, Omega, 53, pp. 49-57.

11. Seeletse, S.M. (2014). A measuring approach in bidding selection procedures for large projects, International Journal of Advances in Management, Economics and Entrepreneurship, 1(1), pp. 7-16.

12. Seeletse, S.M. (2015). Value engineering in SME trade through socially responsible initiatives, International Review of Management and Business Research, 4(4), pp. 1048-1057.

13. Triantaphyllou, E. (2000). Multi-criteria decision making methods: a comparative study. London: Kluwer Academic Publishers.

14. Wang, Y.J. \& Lee, H.S. (2007). Generalizing TOPSIS for fuzzy multiple-criteria group decision-making, Computers and Mathematics with Applications, 53, pp. 1762-1772. 